\title{
Indications for thrombectomy in acute ischemic stroke from emergent large vessel occlusion (ELVO): report of the SNIS Standards and Guidelines Committee
}

\author{
Maxim Mokin, ${ }^{1}$ Sameer A Ansari, ${ }^{2}$ Ryan A McTaggart, ${ }^{3}$ Ketan R Bulsara, ${ }^{4}$ \\ Mayank Goyal, ${ }^{5}$ Michael Chen, ${ }^{6}$ Justin F Fraser, ${ }^{7}$ Society of Neurolnterventional Surgery
}

'Department of Neurosurgery, University of South Florida Tampa, Florida, USA 2Department of Radiology, Neurology, and Neurological Surgery, Northwestern University, Chicago, Illinois, USA ${ }^{3}$ Department of Diagnostic Imaging, Warren Alpert Medical School of Brown University, Providence, Rhode Island, USA ${ }^{4}$ Division of Neurosurgery, University of Connecticut, Farmington, Connecticut, USA ${ }^{5}$ Diagnostic Imaging, University of Calgary, Calgary, Alberta, Canada

${ }^{6}$ Neurological Sciences, Rush University Medical Center, Chicago, Illinois, USA

${ }^{7}$ Neurological Surgery, University of Kentucky, Lexington, Kentucky, USA

\section{Correspondence to}

Dr Justin F Fraser, Departments of Neurological Surgery, Neurology, Radiology, and Neuroscience, University of Kentucky, Lexington KY 40536, USA; Jfr235@uky.edu

Received 5 December 2018 Accepted 11 December 2018 Published Online First 4 January 2019

\section{INTRODUCTION}

This document aims to provide an update on indications for mechanical thrombectomy in acute ischemic stroke (AIS) from emergent large vessel occlusion (ELVO) in the anterior circulation. This reflects new evidence building on the Society of NeuroInterventional Surgery (SNIS) recommendations published in $2015 .{ }^{1}$ Recommendations herein supersede those of previous SNIS guidelines where overlap exists. Previous publications included an overview of prehospital care, a summary of the role of intravenous (IV) and intra-arterial (IA) therapies, a review of technical aspects of thrombectomy, and initial hospital management. ${ }^{1-3}$ This guideline focuses on updated indications for thrombectomy of anterior circulation AIS. Evaluation and management of posterior circulation ELVO will be summarized in separate recommendations.

\section{MATERIALS AND METHODS}

The Standards and Guidelines Committee of the SNIS, a multidisciplinary society representing the leaders in the field of endovascular therapy for neurovascular disease, prepared this document based on a comprehensive review of the available English language literature relating to the topic. Recommendations follow the American College of Cardiology/American Heart Association (ACC) AHA) classification of recommendation/level of evidence and definition of classes and levels of evidence used in American Heart Association/ American Stroke Association (AHA/ASA) recommendations. ${ }^{4}$ Throughout this document, we will refer to the specific selection criteria from the recent randomized trials and registries of modern thrombectomy approaches, which are summarized in table 1 .

\section{INDICATIONS FOR THROMBECTOMY}

Defining the criteria to evaluate and select patients with ELVO for endovascular treatment is critically important, since between $3 \%$ and $22 \%$ of patients with AIS are potentially eligible for mechanical thrombectomy, depending on the specific selection criteria used. $^{5-7}$ Time of symptom onset (or last known well, magnitude of early ischemic change on initial imaging, clinical severity of stroke symptoms, pre-stroke level of functioning and anatomic location of the ELVO are the most important determinants of candidacy for mechanical thrombectomy.

\section{Time from symptom onset}

MR CLEAN, ${ }^{8}$ EXTEND-IA, ${ }^{9}$ and SWIFT PRIME ${ }^{10}$ proved the value of thrombectomy in anterior circulation AIS within the first 6 hours of symptom onset. Specifically, 33\% of patients achieved a good clinical outcome (defined as modified Rankin Scale (mRS) score 0-2) with thrombectomy versus $19 \%$ with medical therapy in MR CLEAN (with an OR of 1.67). In EXTEND-IA, the respective outcomes were $71 \%$ versus $40 \%$ (generalized $\mathrm{OR}=2.0$ ). In SWIFT PRIME, they were $60 \%$ versus $35 \%$ (calculated risk ratio 1.70$)$. THRACE ${ }^{11}$ provided additional data for thrombectomy up to 5 hours $(53 \%$ of patients with thrombectomy achieved a good clinical outcome vs $42 \%$ with medical management, $\mathrm{OR}=1.55)$. The REVASCAT ${ }^{12}$ and ESCAPE ${ }^{13}$ trials provided initial evidence for the benefit of thrombectomy for patients with anterior circulation AIS up to 8 hours ( $44 \%$ with thrombectomy vs $28 \%$ with medical management, adjusted $\mathrm{OR}=2.1$ ) and 12 hours $(53 \%$ rate of good clinical outcome with thrombectomy versus $29 \%$ with medical management, rate ratio 1.8), respectively. A subgroup analysis of 59 patients enrolled within an extended time window (groin puncture within 6-12 hours) in ESCAPE showed similar treatment effects regardless of early and late windows. ${ }^{14}$ A treatment effect favoring thrombectomy was seen across all clinical outcomes.

Two recent multicenter randomized controlled trials (RCTs) of mechanical thrombectomy initiated at a later time windows of up to 16 hours (endovascular therapy following imaging evaluation for ischemic stroke 3 [DEFUSE 3 $]^{15}$ ) and 24 hours from symptom onset (diffusion-weighted imaging [DWI] or CT perfusion [CTP] assessment with clinical mismatch in the triage of wake-up and late presenting strokes undergoing neurointervention with Trevo $[\mathrm{DAWN}]^{16}$ ) have shown that endovascular therapy is safe and highly effective in carefully selected patients with advanced CTP/magnetic resonance (MR) DWI-PWI imaging in comparison with medical management alone. In DEFUSE 3, $45 \%$ of patients achieved good clinical outcome with thrombectomy versus $17 \%$ with medical therapy, OR $=2.67 .{ }^{15}$ In DAWN, there was a similar 
treatment effect with rates of good clinical outcomes of $49 \%$ versus $13 \%$, with an adjusted difference of $33 \% .{ }^{16}$

At first glance, the results of DEFUSE 3 and DAWN suggest a 'late time window paradox' where patients treated with thrombectomy at a later time showed the largest absolute difference in good clinical outcome in comparison with earlier randomized thrombectomy trials. This finding seems to contradict the time-dependency effect of thrombectomy in which each 1 hour delay to reperfusion lowers the chances of good clinical outcome observed in HERMES. ${ }^{17}$ However, a more critical concept illustrated by DEFUSE 3 and DAWN was that collateral flow, in addition to elapsed time, together contribute to duration of tissue viability during ischemia. The use of strict imaging-based selection criteria in DEFUSE 3 and DAWN allowed for the surrogate assessment of collaterals, which now represents an important variable in selection of patients for thrombectomy.

While RCTs are considered 'the gold standard' for establishing clinical evidence, their design and applicability to daily clinical practice can be significantly limited by the trials' selection criteria. Registries provide additional useful information by including a wider range of patients that can potentially serve to validate the findings of RCTs. The TRACK ${ }^{18}$ and NASA $^{19}$ registries assessed real-world experience with thrombectomy without any specific restriction in time from symptom onset to treatment. Both registries showed rates of clinical outcomes comparable to the major RCTs $48 \%$ and $42 \%$ rates of good clinical outcome in TRACK and NASA, respectively). Combined, 33\% of patients from both registries were treated with thrombectomy beyond the first 6 hours, and the safety and efficacy of this treatment was equal to that of treatment performed within $0-6$ hours. ${ }^{20}$ No specific imaging criteria or modalities were required in TRACK and NASA; imaging-based patient selection was left to the discretion of each participating site.

Therefore, there are high-level data from multiple RCTs and registries in support of thrombectomy in appropriately selected patients, up to 24 hours from symptom onset or last known well, including wake-up strokes. There is insufficient evidence to evaluate the safety and efficacy of thrombectomy $>24$ hours from symptom onset. Presently, only limited experience with thrombectomy of anterior circulation ELVO after 24 hours has been reported. ${ }^{21}$

\section{Imaging criteria for thrombectomy}

RCTs have used a number of radiographic selection criteria to determine candidacy for thrombectomy. Specific imaging criteria from these RCTs are summarized in table 1 . The primary purpose of imaging in patient selection is to exclude patients unlikely to benefit or for whom thrombectomy may be harmful. Selection criteria for the late-window trials ( $>6$ hours) were more selective than the $<6$ hours RCTs. For thrombectomy $<6$ hours, there are insufficient data to establish the superiority of one particular imaging modality. These include non-contrast CT, MR DWI, multiphase CT angiography (mCTA) collateral imaging, and advanced CT/MR perfusion imaging with standardized postprocessing techniques. Various imaging criteria such as the Alberta Stroke Programme Early CT Score (ASPECTS) 6-10, MRI DWI ASPECTS 5-10, moderate-to-good collateral status on mCTA ( $>50 \%$ middle cerebral artery [MCA] territory), small $(<50-70 \mathrm{~mL})$ core infarct volumes, and significant penumbra to core mismatch on perfusion imaging have been used as selection criteria in study subjects with anterior circulation stroke and symptom onset up to 6 hours who qualify for thrombectomy.

In patients 6-24 hours, MR DWI-PWI and CTP imaging are highly accurate in selecting patients for thrombectomy when based on criteria used in DEFUSE 3 and DAWN. However, as discussed earlier, both trials, because of restrictive radiographic selection criteria, limit their generalizability to many patients with ELVO. For example, a single-center study of patients presenting 6-24 hours after last known well showed that $70 \%$ of these patients with anterior circulation ELVO were DAWN and/ or DEFUSE 3 ineligible. ${ }^{22}$ Yet, thrombectomy in these patients resulted in rates of good clinical outcomes comparable to those of DAWN and DEFUSE 3 eligible patients. ${ }^{22}$

Data from the early HERMES collaboration of five RCTs, ${ }^{23}$ subgroup analyses of THRACE, ${ }^{24}$ and matched case-control studies indicated that thrombectomy may be beneficial even in patients with ischemic strokes presenting with large core infarct volumes, such as those with CT/MRI ASPECTS $3-5$ or MRI DWI and CTP estimated core volumes of $>50-70 \mathrm{~mL}^{3-25-27}$ The latest HERMES collaboration analysis based on individual patient data from RCTs (MR CLEAN, ESCAPE, EXTEND-IA, SWIFT PRIME, REVASCAT, THRACE, and PISTE) showed that thrombectomy led to better clinical outcomes at 90 days across a broad range of imaging categories, including ASPECTS $<6(\mathrm{OR}=1.58$ for ASPECTS $5-7$ and $\mathrm{OR}=2.15$ for ASPECTS $0-4$ ) and infarcts affecting $>1 / 3$ of MCA territory (OR 1.7), supporting the value of thrombectomy in some patients with large infarcts at baseline. ${ }^{28}$ However, thrombectomy in patients with such unfavorable imaging profiles had higher rates of symptomatic intracranial hemorrhage than in the control group. A separate HERMES collaboration analysis of seven RCTs also confirmed that thrombectomy administered within 0-6hours of symptom onset remained beneficial in patients with a large $(>70 \mathrm{~mL})$ ischemic core measured with CTP or MRI. ${ }^{29}$

\section{Location of large vessel occlusion}

The anatomic definition of an ELVO is variable. ${ }^{30}$ For the purpose of this document, any arterial location or lesion that can be safely selectively catheterized with modern endovascular devices will be referred to as an ELVO. Rigorous clinical trial data support mechanical thrombectomy in patients with intracranial and extracranial occlusions of the internal carotid artery (ICA), including tandem or isolated occlusion of the M1 and M2 segments of the MCA (table 1). Patients with M2 occlusions were also included for thrombectomy in the MR CLEAN, ESCAPE, EXTEND-IA, REVASCAT, and THERAPY trials, although in limited numbers.

The benefit of thrombectomy for more distal MCA occlusions, such as the M3 segments, or anterior cerebral artery occlusions is unclear despite a number of studies evaluating the outcomes of thrombectomy in these patients. This is due to a paucity of data representative of this cohort. Although studies indicate that thrombectomy may be safe and effective in patients with distal anterior circulation ELVO, evidence also suggests an increased risk profile with mechanical interventions, such as vessel perforation and vasospasm. ${ }^{31-34}$ Furthermore, although the natural history of distal occlusions is probably, on average, more favorable than proximal occlusions, the level of disability may still be significant and warrants clarification.

\section{Stroke severity}

When determining eligibility for thrombectomy, stroke severity should be assessed using the National Institutes of Health Stroke Scale (NIHSS). ${ }^{35}$ Use of a standardized scale directly quantifies the degree of neurological deficit, facilitates communication, helps to identify patients for thrombolytic or mechanical intervention, allows objective measurement of changing clinical status, and may identify those at higher risk for complications 


\begin{tabular}{|c|c|c|c|}
\hline Study & Stroke severity & Study groups & Outcomes \\
\hline Haussen et al, $2018^{37}$ & NIHSS $<6$ & $\begin{array}{l}88 \text { Patients with medical therapy, } 30 \text { with } \\
\text { EVT. } \\
\text { Retrospective, matched analysis }\end{array}$ & EVT predicted favorable NIHSS shift $(\beta-3.7,95 \% \mathrm{Cl}-6.0$ to $-1.5, \mathrm{p}=0.02)$. \\
\hline Haussen et al, $2017^{38}$ & NIHSS $<6$ & $\begin{array}{l}22 \text { Patients with medical therapy, } 10 \text { with } \\
\text { EVT }\end{array}$ & $\begin{array}{l}\text { 9/22 (41\%) of medically treated patients deteriorated and required thrombectomy } \\
\text { EVT predicted favorable NIHSS shift } \beta-4.2,95 \% \mathrm{Cl}-8.2 \text { to }-0.1, \mathrm{p}=0.04)\end{array}$ \\
\hline Dargazanli et al, $2017^{40}$ & NIHSS $<8$ & $\begin{array}{l}170 \text { Patients with medical therapy, } 131 \\
\text { with EVT }\end{array}$ & $\begin{array}{l}\text { 24/131 ( } 18 \%) \text { of medical treated patients deteriorated and required EVT. } \\
\text { mRS } 0-1 \text { at } 3 \text { months more common with thrombectomy (adjusted OR=1.79, } \\
95 \% \mathrm{Cl} 1.02 \text { to } 3.14, \mathrm{p}=0.043) \text {. } \\
\text { Similar rates of mRS } 0-2(\mathrm{OR}=1.33,95 \% \mathrm{Cl} 0.71 \text { to } 2.50, \mathrm{p}=0.38)\end{array}$ \\
\hline Heldner et al, $2015^{55}$ & NIHSS $<6$ & 44 Patients treated with medical therapy & $\begin{array}{l}\text { Worsening of NIHSS (increase of score } \geq 1 \text { ) within the first } 24 \text { hours in } 10 / 44(23 \%) \\
\text { Worsening of NIHSS at } 3 \text {-month follow-up in } 17 / 41(41 \%)\end{array}$ \\
\hline Mokin et al, $2014^{39}$ & NIHSS $<8$ & 204 Patients treated with medical therapy & $\begin{array}{l}\text { At discharge, } 17 \% \text { of patients with NIHSS } 0-4 \text { and } 36 \% \text { with NIHSS } 5-7 \text { could not } \\
\text { ambulate independently }\end{array}$ \\
\hline Rajajee et al, $2006^{56}$ & NIHSS $<5$ & 39 Patients treated with medical therapy & $\begin{array}{l}3 / 8(38 \%) \text { patients with ELVO vs } 1 / 31(3 \%) \text { patient without ELVO had early } \\
\text { neurologic deterioration with infarct expansion on MRI (OR=18, } 95 \% \mathrm{Cl} 1.6 \text { to } \\
209, \mathrm{p}=0.02) \text {. }\end{array}$ \\
\hline
\end{tabular}

ELVO, emergent large vessel occlusion; EVT, endovascular therapy; IV, intravenous; MRI, magnetic resonance imaging; mRS, modified Rankin scale; NIHSS, National Institutes of Health Stroke Scale; tPA, tissue plasminogen activator.

such as intracerebral hemorrhage. There was significant variability in the NIHSS score thresholds used for inclusion in recent RCTs for thrombectomy. The MR CLEAN trial had the lowest NIHSS score threshold of 2, ESCAPE and EXTEND-IA set no specific NIHSS score thresholds, but required the symptoms to be 'disabling', whereas other trials had variable ranges from an NIHSS score of 6 to 10 (table 1). Because several trials independently validated an NIHSS threshold of 6 (SWIFT PRIME, REVASCAT and DEFUSE 3), an NIHSS score of $>6$ may serve as a working criterion of clinical severity when considering thrombectomy in anterior circulation AIS. However, patients presenting with anterior circulation ELVO and NIHSS score $<6$, but with otherwise disabling symptoms such as isolated aphasia, should be considered for thrombectomy. Several studies describe a poor natural history in this cohort, with sometimes eventual worsening of neurologic deficits and functional independence in up to a quarter of patients. ${ }^{36}$ More data on this topic are needed (table 2). ${ }^{37-40}$

\section{Thrombectomy and intravenous thrombolysis}

In recent RCTs of thrombectomy, patients eligible for IV thrombolysis with recombinant tissue plasminogen activator (rtPA; alteplase) before thrombectomy received both treatments (table 1). Although there is accumulating evidence suggesting that administering IV rtPA in thrombectomy-eligible patients may be futile, ${ }^{41-43}$ there are also data suggesting that it may be beneficial in patients with ELVO. ${ }^{44}$ This topic is complicated by evidence showing that the efficacy of IV tPA depends on thrombus location; proximal thrombi are less likely to respond to thrombolysis, whereas tPA has a higher likelihood of inducing recanalization at more distally located thrombi. ${ }^{4546}$ Thus, future trials comparing thrombectomy with thrombolysis may need to include analysis by thrombus location. A RCT comparing the safety and efficacy of thrombectomy with rtPA versus thrombectomy alone is currently underway in Europe (SWIFT DIRECT, ClinicalTrials.gov NCT03192332). Additional studies are being considered evaluating lytics other than alteplase in select patients with ELVO with an extended time window. Presently, in patients with anterior circulation ELVO who are eligible for both treatment approaches, IV rtPA should be given. However, treatment with IV rtPA should not delay the initiation of thrombectomy in these patients.

\section{Age and baseline level of functioning}

Significant pre-existing disability often precludes favorable clinical outcomes despite successful thrombectomy. Whether it is due to frailty, poor nutritional status, limited baseline functional capacity, or comorbidities, such patients would, in theory, have almost nothing to gain from thrombectomy. No randomized data exist for thrombectomy in patients with mRS score $>1$, but this may be a topic of interest for future investigators. On the other hand, multiple studies evaluating thrombectomy in elderly patients have showed mixed but promising results. ${ }^{47-49}$ The HERMES collaboration analysis of patient-level data of five RCTs showed significant benefit of thrombectomy in patients aged $>80$ years with a common OR of $3.68 .^{23}$

\section{RECOMMENDATIONS}

\section{Time from symptom onset}

- For anterior circulation AIS, thrombectomy is indicated in select patients up to 16 hours from symptom onset or time last known well for unwitnessed strokes, including wake-up strokes [class I, level A], and is indicated in select patients up to 24 hours from last known normal [class IIa, level B]. This replaces the 2015 SNIS guidelines in which thrombectomy recommendations were limited to the first 6 hours of symptom onset.

\section{Imaging}

- In patients with anterior circulation AIS within the first 6 hours of symptom onset and either CT ASPECTS $\geq 6$, MRI DWI ASPECTS $\geq 6$, moderate-to-good collateral status on mCTA ( $>50 \%$ MCA territory), small $(<50-70 \mathrm{~mL})$ core infarct volumes, and/or significant penumbral to core mismatch on advanced perfusion imaging (CTP or MRI-DWI-PWI), thrombectomy is indicated [class I, level A].

- Thrombectomy may be reasonable within the first 6 hours of symptom onset in patients with a large core infarct volume such as CT ASPECTS of $<6$, MRI DWI or CTP-estimated core volume $>70 \mathrm{~mL}$ [class IIa, level A].

- In patients with anterior circulation AIS due to intracranial ICA and/or M1 occlusion within 6-24hours of symptom onset who meet the advanced MRI DWI-PWI or CTP 
imaging criteria for DAWN or DEFUSE 3, thrombectomy is indicated [class I, level B].

- Thrombectomy may be indicated in carefully selected patients with anterior circulation AIS within 6-24 hours of symptom onset who do not meet imaging criteria for DAWN and DEFUSE 3 but otherwise have a 'favorable' imaging profile such as CT ASPECTS of 6-10, MRI DWI ASPECTS of 6-10, moderate-to-good collateral status on mCTA, or small $(<70 \mathrm{~mL})$ core infarct on advanced MRI DWI-PWI or CTP imaging [class IIb, level B-NR (non-randomized)].

\section{Location of ELVO}

- Thrombectomy is indicated in patients with occlusions of the ICA (including intracranial, cervical segments or tandem occlusion) and M1/M2 MCA [class I, level A]. This replaces the 2015 SNIS guidelines in which thrombectomy recommendations were limited to ICA and MCA M1 LVO locations.

- The benefit of thrombectomy in more distal segments, such as MCA M3 or anterior cerebral artery is unclear. Thrombectomy of such patients may be reasonable in some cases and should be considered on a case-by-case basis [class IIb, level B-NR].

\section{Stroke severity}

- Thrombectomy is indicated in patients with anterior circulation ELVO with NIHSS score $\geq 6$ [class I, level A].

- Thrombectomy may be considered in patients with anterior circulation AIS and NIHSS score $<6$ when associated with disabling symptoms [class IIa, level B-NR]. However, care should be taken when treating these patients to keep complication and hemorrhagic rates below those reported in RCTs.

\section{Age and baseline level of functioning}

- Age $>80$ years should not be used as a contraindication for thrombectomy [class IIa, level A].

- The benefit of thrombectomy in patients with baseline mRS score $>1$ is unknown.

Collaborators AS Arthur, B Baxter, G Dabus, D Frei, CD Gandhi, S Hetts, MS Hussain, MV Jayaraman, P Kan, Y Kayan, RP Klucznik, SK Lee, WJ Mack, T LeslieMazwi, PM Meyers, J Milburn, J Mocco, AT Patsalides, CJ Prestigiacomo, GL Pride, Jr., RM Starke, PJ Sunenshine, G Toth

Contributors MM was the primary author and, as such, was responsible for overseeing construction of the document outline, gathering of evidence, and drafting of the manuscript. He also contacted our guest author (MG) as a topic expert, and sought his input. SAA, RAM, and KRB all made major contributions to the manuscript draft, and supplemented entire sections of the text. They also participated in discussions about the outline and organization of the text. MC acted as a representative of the Board of the Society of Neurolnterventional Surgery, and provided commentary and editing on behalf of the Board and of the Society as a whole. MG was the invited topic expert, whose knowledge and experience in venous thrombosis disease provided a vital international perspective. JFF, as senior author, was responsible for organizing the writing group, overseeing the outline construction, facilitating communication between the writing group and the Standards and Guidelines Committee, drafting and editing the manuscript, and the submission/proofing for publication.

Funding The authors have not declared a specific grant for this research from any funding agency in the public, commercial or not-for-profit sectors.

Disclaimer This literature review ('Review') is provided for informational and educational purposes only. Adherence to any recommendations included in this Review will not ensure successful treatment in every situation. Furthermore, the recommendations contained in this Review should not be interpreted as setting a standard of care, or be deemed inclusive of all proper methods of care or exclusive of other methods of care reasonably directed to obtaining the same results. The ultimate judgment about the propriety of any specific therapy must be made by the physician and the patient in light of all the circumstances presented by the individual patient, and the known variability and biological behavior of the medical condition. This Review and its conclusions and recommendations reflect the best available information at the time it was prepared. The results of future studies may require revisions to the recommendations in this Review to reflect new data. SNIS does not warrant the accuracy or completeness of the Review and assumes no responsibility for any injury or damage to people or property arising out of, or related to, any use of this Review or for any errors or omissions.

Competing interests JFF is an equity interest holder for Fawkes Biotechnology, LLC, and a consultant for Stream Biomedical and for Medtronic. MM is a consultant for Toshiba (Canon) Medical, Penumbra, and Cerebrotech. MG is a consultant for Medtronic, Stryker, Microvention, and Cerenovus. He has a licensing agreement with GE Healthcare for systems of stroke diagnosis. Stryker has provided an unrestricted research grant tothe University of Calgary. MC is a consultant for Medtronic, Stryker, Penumbra, Genentech, and GE.

Patient consent Not required.

Provenance and peer review Not commissioned; internally peer reviewed.

Data sharing statement Not applicable.

\section{REFERENCES}

1 Jayaraman MV, Hussain MS, Abruzzo T, et al. Embolectomy for stroke with emergent large vessel occlusion (ELVO): report of the Standards and Guidelines Committee of the Society of Neurolnterventional Surgery. J Neurointerv Surg 2015;7:316-21.

2 Gandhi CD, Al Mufti F, Singh IP, et al. Neuroendovascular management of emergent large vessel occlusion: update on the technical aspects and standards of practice by the Standards and Guidelines Committee of the Society of Neurolnterventional Surgery. J Neurointerv Surg 2018;10:315-20.

3 Pride GL, Fraser JF, Gupta R, et al. Prehospital care delivery and triage of stroke with emergent large vessel occlusion (ELVO): report of the Standards and Guidelines Committee of the Society of Neurointerventional Surgery. J Neurointerv Surg 2017:9:802-12.

4 Morrison LJ, Gent LM, Lang E, et al. Part 2: Evidence evaluation and management of conflicts of interest: 2015 American Heart Association Guidelines update for cardiopulmonary resuscitation and emergency cardiovascular care. Circulation 2015;132(Suppl 2):S368-82.

5 Mokin M, Pendurthi A, Ljubimov V, et al. ASPECTS large vessel occlusion, and time of symptom onset: estimation of eligibility for endovascular therapy. Neurosurgery 2018;83:122-7.

6 Rai AT, Seldon AE, Boo S, et al. A population-based incidence of acute large vessel occlusions and thrombectomy eligible patients indicates significant potential for growth of endovascular stroke therapy in the USA. J Neurointerv Surg 2017;9:722-6.

7 Vanacker P, Lambrou D, Eskandari A, et al. Eligibility and predictors for acute revascularization procedures in a stroke center. Stroke 2016;47:1844-9.

8 Berkhemer OA, Fransen PS, Beumer D, et al. A randomized trial of intraarterial treatment for acute ischemic stroke. N Engl J Med 2015;372:11-20.

9 Campbell BC, Mitchell PJ, Kleinig TJ, et al. Endovascular therapy for ischemic stroke with perfusion-imaging selection. N Eng/ J Med 2015;372:1009-18.

10 Saver JL, Goyal M, Bonafe A, et al. Stent-retriever thrombectomy after intravenous t-PA vs. t-PA alone in stroke. N Engl J Med 2015;372:2285-95.

11 Bracard S, Ducrocq X, Mas JL, et al. Mechanical thrombectomy after intravenous alteplase versus alteplase alone after stroke (THRACE): a randomised controlled trial. Lancet Neurol 2016;15:1138-47.

12 Jovin TG, Chamorro A, Cobo E, et al. Thrombectomy within 8 hours after symptom onset in ischemic stroke. N Engl J Med 2015;372:2296-306.

13 Goyal M, Demchuk AM, Menon BK, et al. Randomized assessment of rapid endovascular treatment of ischemic stroke. N Engl J Med 2015;372:1019-30.

14 Evans JW, Graham BR, Pordeli P, et al. Time for a time window extension: insights from late presenters in the ESCAPE trial. AJNR Am J Neuroradio/ 2018;39:102-6.

15 Albers GW, Marks MP, Kemp S, et al. Thrombectomy for stroke at 6 to 16 hours with selection by perfusion imaging. N Engl J Med 2018;378:708-18.

16 Nogueira RG, Jadhav AP, Haussen DC, et al. Thrombectomy 6 to 24 hours after stroke with a mismatch between deficit and infarct. N Engl J Med 2018;378:11-21.

17 Saver JL, Goyal M, van der Lugt A, et al. Time to treatment with endovascular thrombectomy and outcomes from ischemic stroke: a meta-analysis. JAMA 2016;316:1279-88.

18 Zaidat 00, Castonguay AC, Nogueira RG, et al. TREVO stent-retriever mechanical thrombectomy for acute ischemic stroke secondary to large vessel occlusion registry. J Neurointerv Surg 2018;10:516-24.

19 Zaidat 00, Castonguay AC, Gupta R, et al. North American Solitaire stent retriever acute stroke registry: post-marketing revascularization and clinical outcome results. J Neurointerv Surg 2014;6:584-8.

20 Mokin M, Abou-Chebl A, Castonguay AC, et al. Real-world stent retriever thrombectomy for acute ischemic stroke beyond 6 hours of onset: analysis of the NASA and TRACK registries. J Neurointerv Surg 2018:neurintsurg-2018-014272.

21 Desai SM, Haussen DC, Aghaebrahim A, et al. Thrombectomy 24 hours after stroke: beyond DAWN. J Neurointerv Surg 2018;10:1039-42. 
22 Desai SM, Rocha M, Molyneaux BJ, et al. Thrombectomy 6-24 hours after stroke in trial ineligible patients. J Neurointerv Surg 2018;10:1033-7.

23 Goyal M, Menon BK, van Zwam WH, et al. Endovascular thrombectomy after large-vessel ischaemic stroke: a meta-analysis of individual patient data from five randomised trials. The Lancet 2016;387:1723-31.

24 Gautheron V, Xie Y, Tisserand M, et al. Outcome after reperfusion therapies in patients with large baseline diffusion-weighted imaging stroke lesions: a THRACE trial (Mechanical Thrombectomy After Intravenous Alteplase Versus Alteplase Alone After Stroke) subgroup analysis. Stroke 2018;49:750-3.

25 Rebello LC, Bouslama M, Haussen DC, et al. Endovascular treatment for patients With acute stroke who have a large ischemic core and large mismatch imaging profile. JAMA Neurol 2017;74:34-40.

26 Mourand I, Abergel E, Mantilla D, et al. Favorable revascularization therapy in patients with ASPECTS $\leq 5$ on DWI in anterior circulation stroke. J Neurointerv Surg 2018;10:5-9.

27 Gilgen MD, Klimek D, Liesirova KT, et al. Younger stroke patients with large pretreatment diffusion-weighted imaging lesions may benefit from endovascular treatment. Stroke 2015;46:2510-6.

28 Román LS, Menon BK, Blasco J, et al. Imaging features and safety and efficacy of endovascular stroke treatment: a meta-analysis of individual patient-level data. Lancet Neurol 2018;17:895-904.

29 Campbell BCV, Majoie C, Albers GW, et al. HERMES collaborators. Penumbral imaging and functional outcome in patients with anterior circulation ischaemic stroke treated with endovascular thrombectomy versus medical therapy: a meta-analysis of individual patient-level data. Lancet Neurol 2019;18:46-55.

30 Leslie-Mazwi T, Chandra RV, Baxter BW, et al. ELVO: an operational definition. Neurointerv Surg 2018;10:507-9.

31 Altenbernd J, Kuhnt 0 , Hennigs $\mathrm{S}$, et al. Frontline ADAPT therapy to treat patients with symptomatic M2 and M3 occlusions in acute ischemic stroke: initial experience with the Penumbra ACE and 3MAX reperfusion system. J Neurointerv Surg 2018;10:434-9.

32 Premat K, Bartolini B, Baronnet-Chauvet $F$, et al. Single-center experience using the 3 max reperfusion catheter for the treatment of acute ischemic stroke with distal arterial occlusions. Clin Neuroradiol 2018;28:553-62.

33 Haussen DC, Lima A, Nogueira RG. The Trevo XP $3 \times 20$ mm retriever ('Baby Trevo') for the treatment of distal intracranial occlusions. J Neurointerv Surg 2016;8:295-9.

34 Mokin M, Fargen KM, Primiani CT, et al. Vessel perforation during stent retriever thrombectomy for acute ischemic stroke: technical details and clinical outcomes. $J$ Neurointerv Surg 2017;9:922-8.

35 Brott T, Adams HP, Olinger CP, et al. Measurements of acute cerebral infarction: a clinical examination scale. Stroke 1989;20:864-70.

36 Cerejo R, Cheng-Ching E, Hui F, et al. Treatment of patients with mild acute ischemic stroke and associated large vessel occlusion. J Clin Neurosci 2016;30:60-4.

37 Haussen DC, Lima FO, Bouslama M, et al. Thrombectomy versus medical management for large vessel occlusion strokes with minimal symptoms: an analysis from STOPStroke and GESTOR cohorts. J Neurointerv Surg 2018;10:325-9.

38 Haussen DC, Bouslama M, Grossberg JA, et al. Too good to intervene? Thrombectomy for large vessel occlusion strokes with minimal symptoms: an intention-to-treat analysis. J Neurointerv Surg 2017:9:917-21.

39 Mokin M, Masud MW, Dumont TM, et al. Outcomes in patients with acute ischemic stroke from proximal intracranial vessel occlusion and NIHSS score below 8. J Neurointerv Surg 2014;6:413-7.
40 Dargazanli C, Arquizan C, Gory B, et al. Mechanical thrombectomy for minor and mild stroke patients harboring large vessel occlusion in the anterior circulation: a multicenter cohort study. Stroke 2017;48:3274-81.

41 Broeg-Morvay A, Mordasini P, Bernasconi C, et al. Direct mechanical intervention versus combined intravenous and mechanical intervention in large artery anterior circulation stroke: a matched-pairs analysis. Stroke 2016;47:1037-44.

42 Weber R, Nordmeyer H, Hadisurya J, et al. Comparison of outcome and interventional complication rate in patients with acute stroke treated with mechanical thrombectomy with and without bridging thrombolysis. J Neurointerv Surg 2017;9:229-33

43 Coutinho JM, Liebeskind DS, Slater LA, et al. Combined intravenous thrombolysis and thrombectomy vs thrombectomy alone for acute ischemic stroke: a pooled analysis of the SWIFT and STAR studies. JAMA Neurol 2017;74:268-74.

44 Goyal N, Tsivgoulis G, Frei D, et al. Comparative safety and efficacy of combined IVT and MT with direct MT in large vessel occlusion. Neurology 2018;90:e1274-82.

45 Seners P, Turc G, Maïer B, et al. Incidence and predictors of early recanalization after intravenous thrombolysis: a systematic review and meta-analysis. Stroke 2016;47:2409-12.

46 Menon BK, Al-Ajlan FS, Najm M, et al. Association of clinical, imaging, and thrombus characteristics with recanalization of visible intracranial occlusion in patients with acute ischemic stroke. JAMA 2018:320:1017-26.

47 Alawieh A, Chatterjee A, Feng W, et al. Thrombectomy for acute ischemic stroke in the elderly: a 'real world' experience. J Neurointerv Surg 2018;10:1209-17.

48 Son S, Kang DH, Hwang YH, et al. Efficacy, safety, and clinical outcome of modern mechanical thrombectomy in elderly patients with acute ischemic stroke. Acta Neurochir 2017;159:1663-9.

49 Le Bouc R, Clarençon F, Meseguer E, et al. Efficacy of endovascular therapy in acute ischemic stroke depends on age and clinical severity. Stroke 2018;49:1686-94.

50 Mocco J, Zaidat 00, von Kummer R, et al. Aspiration thrombectomy after intravenous alteplase versus intravenous alteplase alone. Stroke 2016;47:2331-8.

51 Lapergue B, Blanc R, Gory B, et al. Effect of endovascular contact aspiration vs stent retriever on revascularization in patients with acute ischemic stroke and large vessel occlusion: the ASTER randomized clinical trial. JAMA 2017:318:443-52.

52 Nogueira RG, Frei D, Kirmani JF, et al. Safety and efficacy of a 3-dimensional stent retriever with aspiration-based thrombectomy vs aspiration-based thrombectomy alone in acute ischemic stroke intervention: a randomized clinical trial. JAMA Neurol 2018;75:304.

53 Mueller-Kronast NH, Zaidat 00, Froehler MT, et al. Systematic evaluation of patients treated with neurothrombectomy devices for acute ischemic stroke: primary results of the STRATIS registry. Stroke 2017;48:2760-8.

54 Pereira VM, Gralla J, Davalos A, et al. Prospective, multicenter, single-arm study of mechanical thrombectomy using Solitaire Flow Restoration in acute ischemic stroke. Stroke 2013:44:2802-7.

55 Heldner MR, Jung S, Zubler C, et al. Outcome of patients with occlusions of the internal carotid artery or the main stem of the middle cerebral artery with NIHSS score of less than 5: comparison between thrombolysed and non-thrombolysed patients. J Neurol Neurosurg Psychiatry 2015;86:755-60.

56 Rajajee V, Kidwell C, Starkman S, et al. Early MRI and outcomes of untreated patients with mild or improving ischemic stroke. Neurology 2006;67:980-4. 